\section{Should Prolonged Infusion of ß-Lactams Become Standard of Practice?}

\section{THE "PRO" SIDE}

Clinicians have long debated whether prolonged IV infusion of antimicrobials, including B-lactams, is a more effective administration strategy than intermittent ("standard") infusion. ${ }^{1,2}$ It is a plausible theory: numerous pharmacokinetic/pharmacodynamic studies have shown that prolonged IV infusion of $ß$-lactams optimizes antimicrobial exposure and the activity of these time-dependent agents. ${ }^{3}$ Therefore, administration of ß-lactams by prolonged infusion has the potential to increase efficacy and decrease the development of resistance. ${ }^{4}$ Furthermore, prolonged infusion may attain target pharmacokinetic/pharmacodynamic exposures with similar or lower cumulative daily doses than those used for intermittent infusion. ${ }^{3}$ In turn, the ability to decrease overall direct consumption of antimicrobials has the potential to reduce antimicrobial-related adverse effects and costs. ${ }^{3}$ Optimization of antimicrobial dosing regimens on the basis of pharmacokinetic/pharmacodynamic data has been recommended by the Infectious Diseases Society of America (IDSA) as a potential antimicrobial stewardship intervention that may improve patient outcomes. ${ }^{5}$

When this topic was last debated in the Canadian Journal of Hospital Pharmacy, in 2010, ${ }^{1,2}$ the clinical evidence available did not convincingly show realization of any of these potential benefits. Combining the uncertainty of the benefits with the logistics required to implement such a strategy, prolonged IV infusion of ß-lactams has not been widely adopted. ${ }^{6,7}$ In addition, the body of clinical evidence available in 2010 had limitations: most of the data were retrospective, and there was substantial patient heterogeneity. Therefore, interpretation of the metaanalyses performed was deemed inconclusive. ${ }^{3,8,9}$ The landscape of treatment options has changed since 2010, with more recent, higher-quality evidence both corroborating the potential benefits of prolonged infusion and showing no signals of increase in mortality or harm. ${ }^{10-13}$ We propose that prolonged infusion represents the best strategy for administration of ß-lactams in selected patients.

The Beta Lactam Infusion Group (BLING) investigators of the Australian and New Zealand Intensive Care Society (ANZICS)
Clinical Trials Group have contributed much of the higher-quality evidence for prolonged IV infusion of $B$-lactams in severe sepsis. The BLING trials were specifically designed to overcome the criticisms of prior studies. The BLING I and II trials were multicentre, prospective, and randomized, focusing on patients with severe sepsis. This is the group of patients who are most likely to benefit from augmented dosing strategies because of significant interpatient variability in pharmacokinetic/pharmacodynamic characteristics. ${ }^{10,11}$ The outcomes of interest were described a priori.

The first study, BLING I, compared continuous infusion with intermittent infusion of piperacillin-tazobactam, meropenem, and ticarcillin-clavulanate in 60 patients with severe sepsis. ${ }^{10}$ Patients receiving renal replacement therapy were excluded. The primary end point - the proportion of patients with plasma antibiotic concentrations exceeding the minimum inhibitory concentration on day 3-was much higher in the continuous infusion arm than the intermittent infusion arm $(82 \%$ versus $29 \% ; p=0.001)$. The secondary end point - the proportion of patients with a clinical response by 7-14 days after therapy — was higher in the continuous infusion arm (70\% versus $43 \%$; $p=0.037)$. However, hospital days outside the intensive care unit (ICU-free days) (19.5 versus 17 days) and survival to hospital discharge (90\% versus $80 \%$ ) did not differ significantly between the groups. BLING II followed the same methodology as BLING I, but the 432 patients in BLING II included patients receiving renal replacement therapy. ${ }^{11}$ In contrast to BLING I, the larger BLING II trial did not show any differences between the 2 groups in terms of ICU-free days at day 28, 90-day survival, clinical cure, and organ failure-free days, thus confirming that no harm was conferred by implementing prolonged infusion. The differences in results between BLING I and BLING II were not entirely unexpected; the inclusion in BLING II of patients requiring renal replacement therapy, a group that likely represents a subset of critically ill patients who may be predisposed to poorer outcomes, could have diluted any potential benefits of the prolonged infusion strategy. The third and largest study in the series, BLING III, is currently underway and will examine 90-day mortality in 7000 ICU patients with severe sepsis. ${ }^{14}$

In the Beta-Lactam Infusion in Severe Sepsis (BLISS) trial, ${ }^{13}$ an open-label randomized controlled trial, continuous infusion 
of piperacillin-tazobactam, meropenem, or cefepime had benefits similar to those observed in the BLING I trial. The BLISS trial involved 140 critically ill patients with severe sepsis not receiving renal replacement therapy. ${ }^{13}$ The primary outcome of clinical cure at 14 days after antibiotic cessation was higher in the continuous infusion arm ( $56 \%$ versus $34 \% ; p=0.011)$, as was the number of ventilator-free days (22 versus 14 days; $p<0.043$ ). Furthermore, patients in the continuous infusion arm had better attainment of pharmacokinetic/pharmacodynamic targets. There was no difference in survival between the treatment arms.

The BLING ${ }^{10}{ }^{10}$ BLING II, ${ }^{11}$ and BLISS ${ }^{13}$ trials collectively represent the least heterogeneous and highest-quality evidence available to date. A meta-analysis of pooled patient-level data from these trials showed that patients who received continuous infusion had lower 30-day hospital mortality (20\% versus $26 \%$; relative risk $0.74,95 \%$ confidence interval $0.56-1.00 ; p=0.045) .{ }^{12}$ Furthermore, intermittent IV infusion of ß-lactams was a statistically significant predictor of hospital mortality in this meta-analysis. To put this into context, one life could be saved with a switch from intermittent to prolonged IV infusion of B-lactam in just 15 critically ill patients. This meta-analysis supports the findings of 2 previous meta-analyses suggesting a mortality benefit of prolonged IV infusion of ß-lactams. ${ }^{15,16}$

We advocate extended IV infusion of $ß$-lactams for critically ill patients as a way to help clinicians extend the effectiveness of the existing antimicrobial armamentarium in the battle against multidrug-resistant organisms. Although Canada has lower rates of multidrug-resistant organisms than other countries, this country is not immune to the rise in the rate of carbapenemaseproducing Enterobacteriaceae observed across the world. A recent report indicates that overall rates of carbapenemase-producing Enterobacteriaceae in Canada have increased by 33\% since 2010. ${ }^{17}$ Administering antimicrobials by prolonged infusion is not a panacea for the problem of resistance, but it is a powerful tool that clinicians can use to optimize antimicrobial utilization, in addition to other well-described antimicrobial stewardship interventions.

Health care practitioners and researchers are gaining ground in the development of new antimicrobials as part of the IDSA initiative to make 10 new systemic antibiotics available by $2020{ }^{18}$ However, we stand to lose this ground if we do not also implement strategies to optimize the utilization of currently available resources. With increasing evidence of benefits, no signal of harm, and reports of successful implementation across a variety of settings, ${ }^{19-23}$ it is time to implement prolonged IV infusion of ß-lactams. As health care providers, we have an obligation to support the progress of initiatives that advance patient care and decrease patient harm.

Those who are victorious plan effectively and change decisively. -Sun Tzu

\section{References}

1. Ariano R, Zelenitsky S. Should IV antibiotics be administered by prolonged infusion? The "pro" side. Can J Hosp Pharm. 2010;63(3):246-8.

2. McCormack J. Should IV antibiotics be administered by prolonged infusion? The "con" side. Can J Hosp Pharm. 2010;63(3):248-9.

3. Grupper M, Kuti JL, Nicolau DP. Continuous and prolonged intravenous B-lactam dosing: implications for the clinical laboratory. Clin Microbiol Rev. 2016;29(4):759-72.

4. Roberts JA, Kruger P, Paterson DL, Lipman J. Antibiotic resistance-what's dosing got to do with it? Crit Care Med. 2008;36(8):2433-40.

5. Barlam TF, Cosgrove SE, Abbo LM, MacDougall C, Schuetz AN, Septimus EJ, et al. Implementing an antibiotic stewardship program: guidelines by the Infectious Diseases Society of America and the Society for Healthcare Epidemiology of America. Clin Infect Dis. 2016;62(10):e51-77.

6. Cotta MO, Dulhunty JM, Roberts JA, Myburgh J, Lipman J. Should B-lactam antibiotics be administered by continuous infusion in critically ill patients? A survey of Australia and New Zealand intensive care unit doctors and pharmacists. Int J Antimicrob Agents. 2016;47(6):436-8.

7. George JM, Colton BJ, Rodvold KA. National survey on continuous and extended infusions of antibiotics. Am J Health Syst Pharm. 2012;69(21): 1895-904.

8. Abdul-Aziz MH, Dulhunty JM, Bellomo R, Lipman J, Roberts JA. Continuous beta-lactam infusion in critically ill patients: the clinical evidence. Ann Intensive Care. 2012;2(1):37.

9. Tamma PD, Putcha N, Suh YD, Van Arendonk KJ, Rinke ML. Does prolonged B-lactam infusions improve clinical outcomes compared to intermittent infusions? A meta-analysis and systematic review of randomized, controlled trials. BMC Infect Dis. 2011;11:181.

10. Dulhunty JM, Roberts JA, Davis JS, Webb SA, Bellomo R, Gomersall C, et al. Continuous infusion of beta-lactam antibiotics in severe sepsis: a multicenter double-blind, randomized controlled trial. Clin Infect Dis. 2013; 56(2):236-44.

11. Dulhunty JM, Roberts JA, Davis JS, Webb SA, Bellomo R, Gomersall C, et al. A multicenter randomized trial of continuous versus intermittent ß-lactam infusion in severe sepsis. Am J Respir Crit Care Med. 2015;192(11): 1298-305.

12. Roberts JA, Abdul-Aziz MH, Davis JS, Dulhunty JM, Cotta MO, Myburgh $\mathrm{J}$, et al. Continuous versus intermittent $B$-lactam infusion in severe sepsis. A meta-analysis of individual patient data from randomized trials. Am J Respir Crit Care Med. 2016;194(6):681-91.

13. Abdul-Aziz MH, Sulaiman H, Mat-Nor MB, Rai V, Wong KK, Hasan MS, et al. Beta-Lactam Infusion in Severe Sepsis (BLISS): a prospective, twocentre, open-labelled randomised controlled trial of continuous versus intermittent beta-lactam infusion in critically ill patients with severe sepsis. Intensive Care Med. 2016;42(10):1535-45.

14. Bellomo R. BLING program: what's in the pipeline? [slide presentation]. Melbourne (Australia): ANZIC Research Centre; [cited 2016 Dec 10]. Available from: www.criticalcare.org.za/content/images/11h00_Bellomo_ RB_N_Fri.pdf

15. Falagas ME, Tansarli GS, Ikawa K, Vardakas KZ. Clinical outcomes with extended or continuous versus short-term intravenous infusion of carbapenems and piperacillin/tazobactam: a systematic review and meta-analysis. Clin Infect Dis. 2013;56(2):272-82.

16. Teo J, Liew Y, Lee W, Kwa AL. Prolonged infusion versus intermittent boluses of $B$-lactam antibiotics for treatment of acute infections: a meta-analysis. Int J Antimicrob Agents. 2014;43(5):403-11.

17. Canadian antimicrobial resistance surveillance system report 2016. Ottawa (ON): Public Health Agency of Canada; 2016 [cited 2016 Dec 10]. Available from: https://www.canada.ca/en/public-health/services/publications/drugshealth-products/canadian-antimicrobial-resistance-surveillance-systemreport-2016.html

18. Gilbert DN, Guidos RJ, Boucher HW, Talbot GH, Spellberg B, Edwards JE Jr, et al.; on behalf of the Infectious Diseases Society of America. The $10 \times$ '20 Initiative: pursuing a global commitment to develop 10 new antibacterial drugs by 2020. Clin Infect Dis. 2010;50(8):1081-3.

19. Heinrich LS, Tokumaru S, Clark NM, Garofalo J, Paek JL, Grim SA. Development and implementation of a piperacillin-tazobactam extended infusion guideline. J Pharm Pract. 2011;24(6):571-6. 
20. Schmees PM, Bergman SJ, Strader BD, Metzke ME, Pointer S, Valenti KM. Outcomes of an extended-infusion piperacillin-tazobactam protocol implementation in a community teaching hospital adult intensive care unit. Am J Health Syst Pharm. 2016;73(11 Suppl 3):S100-5.

21. Brunetti L, Poustchi S, Cunningham D, Toscani M, Nguyen J, Lim J, et al. Clinical and economic impact of empirical extended-infusion piperacillintazobactam in a community medical center. Ann Pharmacother. 2015;49(7): 754-60.

22. Nichols KR, Knoderer CA, Cox EG, Kays MB. System-wide implementation of the use of an extended-infusion piperacillin/tazobactam dosing strategy: feasibility of utilization from a children's hospital perspective. Clin Ther. 2012;34(6):1459-65.

23. Nichols KR, Karmire LC, Cox EG, Kays MB, Knoderer CA. Implementing extended-infusion cefepime as standard of care in a children's hospital: a prospective descriptive study. Ann Pharmacother. 2015;49(4):419-26.

Reem Haj-Darrah, BScPhm, PharmD, ACPR

Elizabeth Leung, PharmD, MSCI, BCPS AQID

Department of Pharmacy

St Michael's Hospital

Toronto, Ontario

Competing interests: None declared.

\section{THE “CON" SIDE}

Advances in the understanding of antibiotic pharmacodynamics have led to the design of tailored dosing regimens. A good example is the administration of $B$-lactam antibiotics by prolonged infusion to maximize their time-dependent action. With this approach, B-lactam agents with short half-lives are administered either with the total daily dose delivered over $24 \mathrm{~h}$ as a continuous infusion or with each dose of an intermittent regimen infused over an extended period of hours (extended infusion). Population modelling using Monte Carlo simulations has indicated that prolonged infusion improves the ability to obtain the desired time above the pathogen's minimum inhibitory concentration, often with lower daily doses than are traditionally prescribed. ${ }^{1,2}$

Indeed, the administration of various ß-lactams by prolonged infusion has been shown to improve patient outcomes in certain clinical situations, such as nosocomial pneumonia, critical illness, and infection with bacteria having higher minimum inhibitory concentrations, for which treatment with traditional dosing regimens may not be successful. ${ }^{3-7}$

But should this method of administration be standard practice for B-lactam agents? I would argue no.

To become standard practice, an initiative or activity should be associated with a consistently superior outcome and/or improved patient safety. Neither of these applies to prolonged infusion of ß-lactams.
First and foremost, data supporting a consistent clinical benefit are lacking. It is beyond the scope of this brief commentary to review the plethora of publications related to prolonged infusion of $\beta$-lactams. However, a number of meta-analyses that included critically ill patients-a population thought to benefit most from prolonged infusion - showed no statistically significant differences in patient outcomes such as clinical response, length of stay, and mortality. ${ }^{8-10}$

Similarly, 3 recent, more robust trials did not identify a consistent benefit of prolonged infusion and therefore do not support this dosing approach as a standard of practice. ${ }^{5,11,12}$ In an open-label, randomized study involving 140 patients with severe sepsis in 2 intensive care units (ICUs) in Malaysia, higher clinical cure rates were observed with continuous infusion of B-lactams than with intermittent bolus dosing. However, the 14- and 30-day survival rates did not differ. ${ }^{5}$ Dulhunty and others, ${ }^{11}$ in a multicentre, double-blind study, randomly assigned 432 patients in the ICU with severe sepsis to prolonged infusion or traditional intermittent dosing of 1 of 3 B-lactams. No difference between dosing regimens was observed in the rate of clinical cure, 90-day survival, or number of ICU-free days. ${ }^{11}$ This study did not confirm the higher clinical cure rates found with continuous infusion in an earlier 60-patient study by the same group. ${ }^{13}$ Finally, a single-centre, prospective, open-label study of 367 adults in the ICU with bacterial infection or neutropenic fever, which compared piperacillin-tazobactam administered by intermittent dosing and by extended infusion, showed no difference between groups in the primary outcome of 14-day mortality or in any of the secondary outcomes, save time to defervescence. ${ }^{12}$

This is not to say that a positive effect has not been observed. Meta-analyses by Falagas and others ${ }^{14}$ and Teo and others ${ }^{15}$ showed significant reductions in mortality with the use of prolonged infusion, whereas Lal and others ${ }^{3}$ and Chant and others ${ }^{16}$ identified improved clinical outcomes, but no significant effect on mortality, with the use of prolonged infusion. In a meta-analysis that focused specifically on patients with severe sepsis, ${ }^{17}$ individual patient data from studies previously mentioned $^{5,11,13}$ were analyzed. A significant advantage in terms of 30-day mortality and clinical cure for ß-lactams delivered by continuous infusion was identified, although this mode of administration was not associated with improved clinical cure in the multivariate analysis. ${ }^{17}$

How can this discrepancy in results be explained? There are a number of possible factors. First, there has been variation in the type of studies included in the meta-analyses and in the patient populations included in the original studies. For instance, meta-analyses that also included observational studies, as opposed to being limited to randomized controlled trials, tended to have positive results. ${ }^{14-16}$ Many studies would have included patients who had lower severity of illness and/or were infected with bacteria having lower minimum inhibitory concentrations, for 
which the pharmacodynamic target can be readily achieved with standard dosing regimens. ${ }^{1}$ In addition, the variety of infections, variability in dosing regimens and concomitant antibiotics, small sample size, and low study quality can also explain the variability in results observed.

It has been proposed that delivery of $\beta$-lactams by prolonged infusion may also have a role in limiting antimicrobial resistance; however, evidence to support this theory is lacking. Very few resistant organisms were reported in studies of intermittent versus prolonged-infusion dosing, ${ }^{14}$ and there was no difference between intermittent and extended-infusion dosing in the emergence of strains of Pseudomonas aeruginosa resistant to piperacillintazobactam in an in vitro model. ${ }^{18}$

If the clinical benefits of administering certain $\beta$-lactams by prolonged infusion have not been consistently realized, then patient safety must also be considered. When assessed in individual studies and meta-analyses, prolonged infusion was not associated with a lower incidence of antibiotic-related adverse effects or additional safety benefits. ${ }^{3,59-15}$ On the contrary, a pilot of piperacillin-tazobactam by extended infusion at The Ottawa Hospital identified unexpected safety concerns for subtherapeutic dosing and was stopped as a result. ${ }^{19}$ These concerns arose when, on occasion, the piperacillin-tazobactam q8h extended-infusion regimen was prescribed and/or charted on the medication administration record (MAR) without specification of the 4-h duration of infusion. There were also concerns about missed order clarifications. ${ }^{19}$ Another institution reported incorrect programming of infusion pumps in $10 \%$ of the doses reviewed during implementation of a similar program. ${ }^{20}$ In my opinion, extended infusion should not be a standard means of $B$-lactam delivery in an institution unless physician order-entry processes and electronic MARs are available. Finally, clinicians may not recognize the need for a loading dose when prescribing continuous infusion, which can result in delays in reaching therapeutic serum levels. This presents yet another safety concern. ${ }^{21,22}$

Additional barriers to prolonged infusion becoming standard practice include limitations related to vascular access and inconvenience to mobile patients because of the prolonged duration of infusions, particularly for continuous infusion; added workload for pharmacists (clarifying orders) and for nurses (assessing eligibility for and tolerance of prolonged infusion); and, depending on the type of infusion pump employed, concern about partially administered extended-infusion doses because of tubing residuals. ${ }^{1,19,21,23}$

It may seem ironic that someone who introduced extended infusion of piperacillin-tazobactam as a pilot in her own institution ${ }^{19}$ is taking the "con" side of a debate as to whether prolonged infusion of B-lactams should become standard practice in Canadian hospitals. One motivation for our project was the significant cost savings that could be realized by using less drug per day to achieve similar or better patient outcomes. However, given the availability of lower-priced generic versions of many antibiotics, the cost savings are unlikely to be as pronounced today, and any savings may be offset by the time invested for education, monitoring, and program evaluation.

In summary, the use of optimal dosing regimens to improve the chance of success in treating patients' infections should be standard practice. For some patients, this may mean administering ß-lactams by prolonged infusion. Currently, however, the collective evidence does not support superior clinical outcomes or fewer adverse events with routine use of prolonged infusion for ß-lactams. Thus, the potential for errors, patient inconvenience, increased workload, and limited economic gains, without a clear clinical or safety benefit, should at present relegate the use of prolonged infusion for $\beta$-lactams to the situations and patients that have been shown most likely to benefit.

\section{References}

1. MacVane SH, Kuti JL, Nicolau DP. Prolonging ß-lactam infusion: a review of the rationale and evidence, and guidance for implementation. Int $J$ Antimicrob Agents. 2014;43(2):105-13.

2. Keel RA, Zhanel GG, Zelenitsky S, Nicolau DP. Pharmacodynamic profiling of antimicrobials against gram-negative respiratory isolates from Canadian hospitals. Can J Infect Dis Med Microbiol. 2011;22(4):132-6.

3. Lal A, Jaoude P, El-Solh AA. Prolonged versus intermittent infusion of ß-lactams for the treatment of nosocomial pneumonia: a meta-analysis. Infect Chemother. 2016;48(2):81-90.

4. Lodise TP Jr, Lomaestro B, Drusano GL. Piperacillin-tazobactam for Pseudomonas aeruginosa infections: clinical implications of an extended infusion dosing strategy. Clin Infect Dis. 2007;44(3):357-63.

5. Abdul-Aziz MH, Sulaiman H, Mat-Nor MB, Rai V, Wong KK, Hasan MS, et al. Beta-Lactam Infusion in Severe Sepsis (BLISS): a prospective, twocentre, open-labelled randomised controlled trial of continuous versus intermittent beta-lactam infusion in critically ill patients with severe sepsis. Intensive Care Med. 2016;42(10):1535-45.

6. Taccone FS, Cotton F, Roisin S, Vincent JL, Jacobs F. Optimal meropenem concentrations to treat multidrug-resistant Pseudomonas aeruginosa septic shock. Antimicrob Agents Chemother. 2012;56(4):2129-31.

7. Kuti JL, Moss KM, Nicolau DP, Knauft RF. Empiric treatment of multidrugresistant Burkholderia cepacia lung exacerbation in a patient with cystic fibrosis: application of pharmacodynamic concepts to meropenem therapy. Pharmacotherapy. 2004;24(11):1641-5.

8. Roberts JA, Webb S, Paterson D, Ho KM, Lipman J. A systematic review on clinical benefits of continuous administration of beta-lactam antibiotics. Crit Care Med. 2009;37(6):2071-8.

9. Tamma PD, Putcha N, Suh YD, Van Arendonk KJ, Rinke ML. Does prolonged B-lactam infusions improve clinical outcomes compared to intermittent infusions? A meta-analysis and systematic review of randomized, controlled trials. BMC Infect Dis. 2011;11:181.

10. Shiu J, Wang E, Tejani AM, Wasdell M. Continuous versus intermittent infusions of antibiotics for the treatment of severe acute infections. Cochrane Database Syst Rev. 2013;(3):CD008481.

11. Dulhunty JM, Roberts JA, Davis JS, Webb SA, Bellomo R, Gomersall C, et al. A multicenter randomized trial of continuous versus intermittent B-lactam infusion in severe sepsis. Am J Respir Crit Care Med. 2015; 192(11):1298-305.

12. Fan SY, Shum HP, Cheng WY, Chan YH, Leung SYM, Yan WW. Clinical outcomes of extended versus intermittent infusion of piperacillin/tazobactam in critically ill patients: a prospective clinical trial. Pharmacotherapy. 2017; 37(1):109-19.

13. Dulhunty JM, Roberts JA, Davis JS, Webb SA, Bellomo R, Gomersall C, et al. Continuous infusion of beta-lactam antibiotics in severe sepsis: a multicenter double-blind, randomized controlled trial. Clin Infect Dis. 2013; 56(2):236-44 
14. Falagas ME, Tansarli GS, Ikawa K, Vardakas KZ. Clinical outcomes with extended or continuous versus short-term intravenous infusion of carbapenems and piperacillin/tazobactam: a systematic review and meta-analysis. Clin Infect Dis. 2013;56(2):272-82.

15. Teo J, Liew Y, Lee W, Kwa AL. Prolonged infusion versus intermittent boluses of $ß$-lactam antibiotics for treatment of acute infections: a meta-analysis. Int J Antimicrob Agents. 2014;43(5):403-11.

16. Chant $\mathrm{C}$, Leung A, Friedrich JO. Optimal dosing of antibiotics in critically ill patients by using continuous/extended infusions: a systematic review and meta-analysis. Crit Care. 2013;29:17(6):R279.

17. Roberts JA, Abdul-Aziz MH, Davis JS, Dulhunty JM, Cotta MO, Myburgh $\mathrm{J}$, et al. Continuous versus intermittent B-lactam infusion in severe sepsis. A meta-analysis of individual patient data from randomized trials. Am J Respir Crit Care Med. 2016:194(6):681-91.

18. Felton TW, Goodwin J, O'Connor L, Sharp A, Gregson L, Livermore J, et al. Impact of bolus dosing versus continuous infusion of piperacillin and tazobactam on the development of antimicrobial resistance in Pseudomonas aeruginosa. Antimicrob Agents Chemother. 2013;57(12):5811-9.

19. Zvonar R, Kanji S. Experience with extended infusion of piperacillintazobactam at a teaching hospital [letter]. Can J Hosp Pharm. 2010;63(3): 252-3.

20. Xamplas RC, Itokazu GS, Glowacki RC, Grasso AE, Caquelin C, Schwartz DN. Implementation of an extended-infusion piperacillin-tazobactam program at an urban teaching hospital. Am J Health Syst Pharm. 2010;67(8):622-8
21. De Waele JJ, Lipman J, Carlier M, Roberts JA. Subtleties in practical application of prolonged infusion of $B$-lactam antibiotics. Int I Antimicrob Agents. 2015;45(5):461-3.

22. Rhodes NJ, MacVane SH, Kuti JL, Scheetz MH. Impact of loading doses on the time to adequate predicted beta-lactam concentrations in prolonged and continuous infusion dosing schemes [letter]. Clin Infect Dis. 2014; 59(6):905-7.

23. Lam WJ, Bhowmick T, Gross A, Vanschooneveld TC, Weinstein MP. Using higher doses to compensate for tubing residuals in extended-infusion piperacillin-tazobactam. Ann Pharmacother. 2013;47(6):886-91.

Rosemary Zvonar, BSCPhm, ACPR, FCSHP

Antimicrobial Pharmacy Specialist

Pharmacy Department

The Ottawa Hospital

Ottawa, Ontario

Competing interests: None declared.

\section{CISADL 2016}

\section{The 2016 Canadian Investigational \& Special Access Drug List is now on sale!}

\section{Please use the CSHP Products \& Publications Order Form found online at CSHP.ca to place your}

order: http://www.cshp.ca/productsServices/otherPublications/canadian/nvestigational e.asp

The Canadian Investigational \& Special Access Drug List (CISADL) is a compilation of drugs that are currently not marketed in Canada, and may have investigational or special access status with Health Canada. This list is not endorsed by Health Canada's Special Access Program. For precise information about the regulatory status of a drug, contact the Special Access Program directly. Entries to the list are contributed to by several drug information centres across Canada and by Canadian drug manufacturers. In cases where non-marketed drugs do not appear on the list, foreign references should be consulted. The content of the list includes generic name and strength/concentration, dosage form, pharmacology/therapeutic use, trade and code name, source (manufacturer/distributor), and comments. Available in English only. 